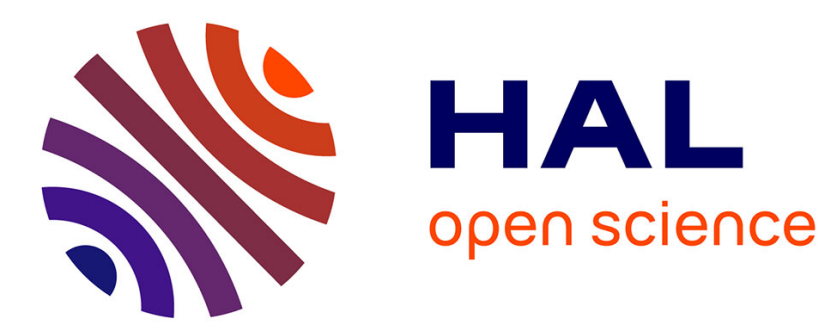

\title{
A High-Order Sliding-Mode Adaptive Observer for Uncertain Nonlinear Systems
}

Héctor Ríos, Roberto Franco, Alejandra Ferreira de Loza, Denis Efimov

\section{To cite this version:}

Héctor Ríos, Roberto Franco, Alejandra Ferreira de Loza, Denis Efimov. A High-Order Sliding-Mode Adaptive Observer for Uncertain Nonlinear Systems. IEEE Transactions on Automatic Control, In press. hal-03503709

\author{
HAL Id: hal-03503709 \\ https://hal.inria.fr/hal-03503709
}

Submitted on 28 Dec 2021

HAL is a multi-disciplinary open access archive for the deposit and dissemination of scientific research documents, whether they are published or not. The documents may come from teaching and research institutions in France or abroad, or from public or private research centers.
L'archive ouverte pluridisciplinaire HAL, est destinée au dépôt et à la diffusion de documents scientifiques de niveau recherche, publiés ou non, émanant des établissements d'enseignement et de recherche français ou étrangers, des laboratoires publics ou privés. 


\title{
A High-Order Sliding-Mode Adaptive Observer for Uncertain Nonlinear Systems
}

\author{
Héctor Ríos ${ }^{\dagger \star}$, Roberto Franco ${ }^{\dagger}$, Alejandra Ferreira de Loza $^{\ddagger \star}$ and Denis Efimov ${ }^{\S *}$
}

\begin{abstract}
A high-order sliding-mode adaptive observer is proposed to solve the problem of adaptive estimation, i.e., the simultaneous estimation of the state and parameters, for a class of uncertain nonlinear systems in the presence of external disturbances, that does not need to satisfy a relative degree condition equal to one. This approach is based on a highorder sliding-mode observer and a nonlinear parameter identification algorithm. The practical, global and uniform asymptotic stability of the adaptive estimation error, despite the external disturbances, is guaranteed through the small-gain theorem. The convergence proofs are developed based on Lyapunov and inputto-state stability theories. Some simulation results illustrate the performance of the proposed high-order sliding-mode adaptive observer.
\end{abstract}

Index Terms-Adaptive Observers, Nonlinear Systems, SlidingModes.

\section{INTRODUCTION}

Control of real systems confronts two shortcomings: first, not all the states are available for measurement; second, the model has discrepancies with respect to the real system (see [1] and [2]). Disturbances and uncertainties exist in almost all realworld scenarios in the form of external perturbations, unknown system dynamics and/or unknown parameters. In the last years, the application and the development of adaptive observers have motivated a lot of work, this topic has become a wide and active research field. The adaptive observers estimate, simultaneously, the whole state and the parameters of the system using some online adaptation law (for instance, see [3]).

Concerning adaptive observers, early results that solve this problem are presented in [4] and [5]. Both works guarantee exponential convergence of the estimation error, meanwhile, the convergence of the parameters is exponential. The latter fact is mainly determined by a persistence-of-excitationtype constraint. The problem of adaptive observer synthesis

This work was supported in part by the SEP-CONACYTECOS-ANUIES Project 315597 and ECOS NORD Project M20M04. The work of Roberto Franco and Héctor Ríos was supported in part by CONACYT CVU 772057, in part by Catedras CONACYT CVU 270504 Project 922, and in part by TecNM Projects. The work of Alejandra Ferreira de Loza was supported by Catedras CONACYT CVU 166403 Project 1537.

†Tecnológico Nacional de México/I.T. La Laguna, División de Estudios de Posgrado e Investigación, C.P. 27000, Torreón, Coahuila, México.

*Cátedras CONACYT, C.P. 03940, Ciudad de México, México.

${ }^{\ddagger}$ Instituto Politécnico Nacional-CITEDI, C.P. 22435, Tijuana, Baja California, México.

§Inria, Univ. Lille, CNRS, UMR 9189-CRISTAL, F-59000, Lille, France.

* Department of Control Systems and Informatics, Information Technologies Mechanics and Optics University, Saint Petersburg 197101, Russia. for Lipschitz nonlinear systems with known parameters is developed in [6]. The sufficient conditions are presented to ensure the state estimation error convergence to zero. Also, an adaptive observer scheme is presented to achieve the adaptive estimation under additional constraints. In [7], based on the concepts of weakly attracting sets and nonuniform convergence, an adaptive observer is proposed for the asymptotic reconstruction of the state and parameter values in a particular class of forward-complete Single-Input-Single-Output nonlinear systems. In [8], an adaptive observer is proposed to exponentially estimate the state and the unknown parameters under a persistent excitation condition for uniformly observable Multiple-Input-Multiple-Output (MIMO) nonlinear systems. In [9], an $L_{1}$ adaptive descriptor observer is designed for multi-variable systems with nonlinear uncertainties and measurement noises. The algorithm is constructed to asymptotically estimate states, nonlinear uncertainties, and measurement noise at the same time. In [10], a robust adaptive observer design methodology for a class of uncertain nonlinear systems in the presence of time-varying unknown parameters is given ensuring asymptotic convergence of state estimation and boundedness of parameter errors. The above-mentioned works do not consider external disturbances, and the convergence rates are asymptotic or exponential.

In this context, in [11], two nonlinear adaptive observers are designed to provide a fault diagnosis of a Solid Oxide Fuel Cell system, ensuring an asymptotic convergence of the fault. In [12], two types of adaptive observer methods are developed to solve the problem of Markovian jump systems with simultaneous time-varying actuator efficiency factors, additive actuator, and sensor faults. The estimation and identification errors for both observers converge asymptotically. In [13], the problem of an adaptive high-gain observer for a class of MIMO non uniformly observable systems with unknown constant parameters is addressed. Exponential convergence is established for the state and parameter estimation errors. In [14], an adaptive sliding-mode disturbance observer is designed to estimate the disturbance in finite time ensuring the convergence to zero of the state estimation error. In [15], an adaptive sliding-mode observer is proposed for position and velocity sensorless controls of a cylindrical brushless DC motor. This algorithm guarantees asymptotic convergence of the estimation errors.

On the other hand, in the sliding-mode area, in [16], an arbitrary order differentiator that converges to the true derivatives of the signal after a finite time independent of the initial differentiator error is presented. This approach switches from a 
uniform differentiator to the classical high-order sliding-mode (HOSM) differentiator providing convergence to zero in finitetime of the differentiation error. In [17], a functional observer for linear systems with unknown inputs is considered. It is shown that when such an observer is not proper, it is still possible to use an HOSM differentiator to construct a proper quasi-functional unknown input observer.

Regarding finite-time convergence, an adaptive state observer for a class of nonlinear systems with unknown parameters is presented in [18]. Under an excitation assumption, the convergence in finite time of the parameter is guaranteed; meanwhile, the state converges asymptotically. In [19], a nonlinear adaptive sliding-mode observer is proposed based on a nonlinear parameter estimation algorithm. The proposed algorithm is included in the structure of a sliding-mode state observer, providing an ultimate bound for the full estimation error and attenuating the effects of the external disturbances concluding finite-time convergence of the identification error and exponential convergence of the estimation error. In [20], an adaptive observer based on [19] is developed, this observer increases the class of systems ensuring finite-time convergence of the parameter identification error and exponential convergence to a neighborhood of the state estimation error.

Most of the above-mentioned works have one of the following shortcomings: either do not consider external disturbances, or the output must satisfy a relative degree condition equal to one with respect to the external disturbances. Moreover, in most cases, the convergence rates are asymptotic or exponential. Motivated for the aforementioned issues, this paper proposes an HOSM adaptive observer for a class of uncertain nonlinear systems affected by external disturbances. In contrast with the previous works, the disturbances can be of a relative degree higher than one. The present approach combines an HOSM observer with a nonlinear parameter identification algorithm. The HOSM observer guarantees finite-time inputto-state stability (FT-ISS) of the state estimation error with respect to the parameter identification error. Whereas, the nonlinear parameter identification algorithm provides FT-ISS of the parameter identification error with respect to the state estimation error. Then, the practical, global, and uniform asymptotic stability of the adaptive estimation error, despite the external disturbances, is guaranteed through the smallgain theorem. The convergence proofs are developed based on Lyapunov and ISS theories.

This manuscript is organized as follows. The problem statement is formulated in section II. The preliminaries are discussed in Section III. The adaptive observer and the main results are presented in Section IV. Simulation results are shown in Section V, followed by the conclusions in Section VI.

\section{A. Notation}

The Euclidean norm of a vector $q \in \mathbb{R}^{n}$ is denoted by $\|q\|$. For a matrix $Q \in \mathbb{R}^{m \times n}$, denotes its smallest singular value $\sigma_{\min }(Q)=\sqrt{\lambda_{\min }\left(Q^{T} Q\right)}$ and its induced norm as $\|Q\|:=\sqrt{\lambda_{\max }\left(Q^{T} Q\right)}=\sigma_{\max }(Q)$, where $\lambda_{\max }$ is the maximum eigenvalue and $\lambda_{\min }$ is the minimum one, $\sigma_{\max }$ is the largest singular value. For a Lebesgue measurable function $u: \mathbb{R}_{\geq 0} \rightarrow \mathbb{R}^{m}$, define the norm $\|u\|_{\left(t_{0}, t_{1}\right)}:=$ ess $\sup _{t \in\left(t_{0}, t_{1}\right)}\|u(t)\|$, then $\|u\|_{\infty}:=\|u\|_{(0,+\infty)}$ and the set of functions $u$ with the property $\|u\|_{\infty}<+\infty$ is denoted as $\mathcal{L}_{\infty}$. For a matrix function $Q: \mathbb{R}_{\geq 0} \rightarrow \mathbb{R}^{m \times n}$, denote $\|Q\|_{\infty}:=\|Q\|_{(0,+\infty)}$. The term $\nabla \bar{V}(x) f(x)$ denotes the directional derivative of a continuously differentiable function $V$ with respect to the vector field $f$ evaluated at any point $x$. Define the function $\lceil a\rfloor^{\gamma}=|a|^{\gamma} \operatorname{sign}(a)$, for any $\gamma \in[0,1)$ and any $a \in \mathbb{R}$.

A continuous function $\alpha: \mathbb{R}_{\geq 0} \rightarrow \mathbb{R}_{\geq 0}$ belongs to class $\mathcal{K}$ if it is strictly increasing and $\alpha(0)=0$; it belongs to class $\mathcal{K}_{\infty}$ if it is also unbounded. A continuous function $\beta: \mathbb{R}_{\geq 0} \times \mathbb{R}_{\geq 0} \rightarrow$ $\mathbb{R}_{\geq 0}$ belongs to class $\mathcal{K} \mathcal{L}$ if, for each fixed $s, \beta(r, s) \in \mathcal{K}$ with respect to $r$, and for each fixed $r, \beta(r, s)$ is decreasing to zero with respect to $s$. Moreover, the function $\beta$ is class $\mathcal{K} \mathcal{L}_{\mathcal{T}}$ if $\beta(r, 0) \in \mathcal{K}_{\infty}, \beta$ is a strictly decreasing function on its second argument $s \in \mathbb{R}_{+}$for any fixed first argument $r \in \mathbb{R}_{+}$and $\beta(r, T)=0$ for each fixed $r \in \mathbb{R}_{+}$for some $0 \leq T<+\infty$.

\section{Problem Statement}

Consider the following class of uncertain nonlinear systems

$$
\begin{aligned}
& \dot{x}=A x+\phi(y, u)+G(t, y, u) \theta+D w(t), \\
& y=C x,
\end{aligned}
$$

where $x \in \mathbb{R}^{n}$ is the state vector, $y \in \mathbb{R}$ is the measurable output, $u \in \mathbb{R}^{m}$ is the control input vector, $\theta \in \mathbb{R}$ is an unknown constant parameter, and $w \in \mathbb{R}$ represents the external disturbances. The matrices $A, C$, and $D$ are known, they have corresponding dimensions, and it is assumed that the triple $(A, C, D)$ is strongly observable, which implies that the relative degree of the output $y$, with respect to the external disturbance $w$, is equal to $n$. The functions $\phi: \mathbb{R} \times \mathbb{R}^{m} \rightarrow \mathbb{R}^{n}$ and $G: \mathbb{R}_{\geq 0} \times \mathbb{R} \times \mathbb{R}^{m} \rightarrow \mathbb{R}^{n}$ are also known, and they ensure uniqueness and existence of solutions for system (1) for all admissible disturbances.

The objective is to provide estimations of the state and the unknown parameter, i.e., $x$ and $\theta$, respectively; only using the information of the output $y$ and attenuating the effects of the external disturbances $w$.

The following assumption is imposed on system (1).

Assumption 1. $\|x\|_{\infty}<+\infty,\|u\|_{\infty}<+\infty,\|w\|_{\infty}<+\infty$, and $\|G(t, y(t), u(t))\|_{\infty}<+\infty$ for all $t \geq 0$.

\section{PRELIMINARIES}

Consider the following nonlinear system

$$
\dot{x}=f(t, x(t)), t \geq t_{0}, t_{0} \in \mathbb{R},
$$

where $x \in \mathbb{R}^{n}$ is the state vector; $f: \mathbb{R}_{+} \times \mathbb{R}^{n} \rightarrow \mathbb{R}^{n}$ is a continuous function with respect to $x$ and measurable with respect to $t$, and $f(t, 0)=0$. A solution of system (2), for an initial condition $x_{0} \in \mathbb{R}^{n}$ at time instant $t_{0} \in \mathbb{R}$, is denoted as $x\left(t, t_{0}, x_{0}\right)$, and it is defined on some finite time interval $\left[t_{0}, t_{0}+T\right]$, where $0 \leq T \leq \infty$.

Let $\Psi$ be an open neighborhood of the origin in $\mathbb{R}^{n}$; then, the following stability properties are introduced for system (2) (for more details see [19], [21], [22] and [23]). 
Definition 1. [23]. At the steady state $x=0$, the system (2) is said to be:

1) Uniformly Stable in $\Psi$ if, for any $t_{0} \in \mathbb{R}$ and $\epsilon>0$, there is $\delta(\epsilon)>0$ such that for any $x_{0} \in \Psi$, if $\left\|x_{0}\right\|<\delta(\epsilon)$, then $\left\|x\left(t, t_{0}, x_{0}\right)\right\| \leq \epsilon$, for all $t \geq t_{0}$;

2) Uniformly Asymptotically Stable if it is Uniformly Stable in $\Psi$ and for any $t_{0} \in \mathbb{R}, \kappa>0$ and $\epsilon>0$, there exists $T(\kappa, \epsilon) \geq 0$ such that for any $x_{0} \in \Psi$, if $\left\|x_{0}\right\| \leq \kappa$, then $\left\|x\left(t, t_{0}, x_{0}\right)\right\| \leq \epsilon$, for all $t \geq t_{0}+T(\kappa, \epsilon)$;

3) Uniformly Finite-Time Stable if it is Uniformly Stable and for any $x_{0} \in \Psi$, there exists $0 \leq T^{x_{0}}<+\infty$ such that $x\left(t, t_{0}, x_{0}\right)=0$, for all $t \geq t_{0}+T^{x_{0}}$. The function $T\left(x_{0}\right)=\inf \left\{T^{x_{0}} \geq 0: x\left(t, t_{0}, x_{0}\right)=0, \forall t \geq t_{0}+T^{x_{0}}\right\}$ is called the settling time of the system (2).

If $\Psi=\mathbb{R}^{n}$, at steady-state $x=0$, system (2) is said to be Globally Uniformly Stable (GUS), Globally Uniformly Asymptotically Stable (GUAS) and Globally Uniformly Finite-Time Stable (GUFTS), respectively.

Consider the following nonlinear system

$$
\dot{x}=f(x, w),
$$

where $x \in \mathbb{R}^{n}$ is the state vector, $w \in \mathbb{R}^{l}$ is the external disturbance; $f: \mathbb{R}^{n} \times \mathbb{R}^{l} \rightarrow \mathbb{R}^{n}$ is a locally Lipschitz function. It is assumed that the solution of system (3) for an initial condition $x_{0} \in \mathbb{R}^{n}$ and $w \in \mathcal{L}_{\infty}$ is denoted as $x\left(t, x_{0}, w\right)$ for any $t \geq 0$ for which the solution exists.

Definition 2. [21]. The system (3) is said to be Input-to-State practically Stable (ISpS) if for any $w \in \mathcal{L}_{\infty}$, and any $x_{0} \in \mathbb{R}^{n}$ there exist some functions $\beta \in \mathcal{K} \mathcal{L}, \gamma \in \mathcal{K}$ and a constant $\kappa \in \mathbb{R}_{\geq 0}$ such that its solution satisfies

$$
\left\|x\left(t, x_{0}, w\right)\right\| \leq \beta\left(\left\|x_{0}\right\|, t\right)+\gamma\left(\|w\|_{\infty}\right)+\kappa, \forall t \geq 0 .
$$

If $\kappa=0$, the system (3) is said to be Input-to-State Stable (ISS). Moreover, if $\beta \in \mathcal{K} \mathcal{L}_{\mathcal{T}}$ and $\kappa=0$, system (3) is said to be Finite-Time Input-to-State Stable (FT-ISS).

If $\|w\|_{\infty}=0$; then, system (3) is said to be practically $G U A S$, for $\kappa>0$; and GUAS, for $\kappa=0$.

Lemma 1. [19]. Let $V: \mathbb{R}^{n} \rightarrow \mathbb{R}_{\geq 0}$ be a smooth function. If there exist some positive constants $\varphi_{1}, \varphi_{2}, \varphi_{3}, \varphi_{4}>0$ and $\varsigma \in(0,1]$ such that

$$
\begin{gathered}
\varphi_{1}\|x\|^{2} \leq V(x) \leq \varphi_{2}\|x\|^{2} \\
\nabla V(x) f(x, \omega) \leq-\varphi_{3} V^{\varsigma}(x), \forall\|x\| \geq \mu:=\varphi_{4}\|w\|_{\infty},
\end{gathered}
$$

then system (3) is FT-ISS with respect to the input $w$. Moreover, the following bounds are satisfied:

$$
\begin{aligned}
& \left\|x\left(t, x_{o}, \omega\right)\right\| \leq\left\{\begin{array}{l}
\frac{\varphi(t)}{\varphi_{1}}, \forall \varsigma \in(0,1), \forall t \leq T\left(x_{0}\right), \\
e^{-\frac{\varphi_{3}}{2} t} \sqrt{\frac{\varphi_{2}}{\varphi_{1}}}\left\|x_{0}\right\|, \varsigma=1, \quad \forall t \leq T\left(x_{0}\right),
\end{array}\right. \\
& \left\|x\left(t, x_{o}, \omega\right)\right\| \leq \sqrt{\frac{\varphi_{2}}{\varphi_{1}}} \mu, \forall t>T\left(x_{0}\right),
\end{aligned}
$$

where $\varphi(t)=\left(\varphi_{2}^{1-\varsigma}\left\|x_{0}\right\|^{2(1-\varsigma)}-\varphi_{3}(1-\varsigma) t\right)^{\frac{1}{2(1-\varsigma)}}$ and

$$
T\left(x_{0}\right) \leq\left\{\begin{array}{l}
\max \left(0, \frac{\varphi_{2}^{1-\varsigma}\left\|x_{0}\right\|^{2(1-\varsigma)}-\varphi_{1}^{1-\varsigma} \mu^{2(1-\varsigma)}}{\varphi_{3}(1-\varsigma)}\right), \forall \varsigma \in(0,1), \\
\max \left(0, \frac{2\left[\ln \left(\left\|x_{0}\right\|\right)-\ln (\mu)\right]}{\varphi_{3}}\right), \varsigma=1 .
\end{array}\right.
$$

Let us consider the following interconnected nonlinear system

$$
\begin{aligned}
& \dot{x}_{1}=f_{1}\left(x_{1}, x_{2}, w\right), \\
& \dot{x}_{2}=f_{2}\left(x_{1}, x_{2}, w\right),
\end{aligned}
$$

where $x_{i} \in \mathbb{R}^{n_{i}}, w \in \mathbb{R}^{l}$, and $f_{i}: \mathbb{R}^{n_{1}} \times \mathbb{R}^{n_{2}} \times \mathbb{R}^{l} \rightarrow \mathbb{R}^{n_{i}}$ ensures existence of the system solutions at least locally, for $i=1,2$. Consider that both subsystems are ISS with respect to $\left(x_{2}, w\right)$ and $\left(x_{1}, w\right)$, respectively, i.e., there exist functions $\beta_{i} \in \mathcal{K} \mathcal{L}, \gamma_{j} \in \mathcal{K}$, and positive constants $\kappa_{i}$ with $i=1,2$ and $j=\overline{1,4}$, such that the conditions

$$
\begin{aligned}
\left\|x_{1}\left(t, x_{10}, w\right)\right\| \leq \beta_{1}\left(\left\|x_{10}\right\|, t\right)+ & \gamma_{1}\left(\left\|x_{2}\right\|_{\infty}\right) \\
& +\gamma_{2}\left(\|w\|_{\infty}\right)+\kappa_{1}, \\
\left\|x_{2}\left(t, x_{20}, w\right)\right\| \leq \beta_{2}\left(\left\|x_{20}\right\|, t\right)+ & \gamma_{3}\left(\left\|x_{1}\right\|_{\infty}\right) \\
& +\gamma_{4}\left(\|w\|_{\infty}\right)+\kappa_{2},
\end{aligned}
$$

hold for all $t \geq 0$. Thus, the following nonlinear smallgain result is introduced, in terms of ISS properties, for the interconnected system (4).

Theorem 1. [24], [25]. Suppose that the interconnected system (4a)-(4b) is ISS with respect to $\left(x_{2}, w\right)$ and $\left(x_{1}, w\right)$, respectively, satisfying the condition in (5). If there exists a non negative number $s_{1}$ satisfying

$$
\gamma_{1} \circ \gamma_{3}(s)<s, \forall s>s_{1},
$$

then, the system (4) is ISpS with respect to the external input $w$. If $s_{1}=0$, the system (4) is ISS with respect to the external input $w$.

Note that, if the external disturbance $w$ is vanishing, or somehow, it is completely compensated, Theorem 1 implies that the interconnected system (4a)-(4b) is practically GUAS, for $s_{1}>0$; and $G U A S$, for $s_{1}=0$.

\section{HOSM ADAPTIVE OBSERVER}

Let us introduce the following adaptive observer

$$
\begin{aligned}
\dot{\Omega} & =(A-L C) \Omega+G(t, y, u), \\
\dot{\hat{\theta}} & =\Gamma \Omega^{T} C^{T}\lceil y-C \hat{x}\rfloor^{\alpha}, \\
\hat{x} & =z+T^{-1} v, \\
\dot{z} & =A z+\phi(y, u)+G(t, y, u) \hat{\theta}+L(y-C z)+\Omega \dot{\hat{\theta}}, \\
\dot{v} & =A_{0} v+a(y-C z)+\chi\left(v_{1}, y-C z\right) .
\end{aligned}
$$

where $\hat{x} \in \mathbb{R}^{n}$ and $\hat{\theta} \in \mathbb{R}$ are the estimations for $x$ and $\theta$, respectively; $z \in \mathbb{R}^{n}$ is a Luenberger state estimation, and $\Omega \in \mathbb{R}^{n}$ is an auxiliary variable. If the signal $G$ is persistently exciting (PE), then, due to the filtering property of the variable $\Omega$, the variable $C \Omega$ is also PE. The function $\lceil y-C \hat{x}\rfloor^{\alpha}$ in (7b) is understood in the component-wise sense. Note that (7b) represents the dynamics of a parameter identification algorithm characterized by $\alpha$. If $\alpha=0$, a discontinuous algorithm is obtained; when $\alpha=1$, one has the classic linear algorithm, while for $\alpha \in(0,1)$, a continuous nonlinear algorithm is given. The observer vector gain $L \in \mathbb{R}^{n}$ has to be selected such that $(A-L C)$ is Hurwitz and 
$\Gamma=\Gamma^{T}>0$. The transformation matrix $T \in \mathbb{R}^{n \times n}$ is given as $T^{-1}=\left[(A-L C)^{n-1} \vartheta,(A-L C)^{n-2} \vartheta, \ldots, \vartheta\right]$; where $\vartheta=\mathcal{O}^{-1} h$, with $h=[0,0, \ldots, 1]^{T} \in \mathbb{R}^{n}$, and $\mathcal{O}$ is the observability matrix of the pair $(A-L C, C)$. The vector $v \in \mathbb{R}^{n}$ provides an estimation of the error between the system state $x$ and the Luenberger state estimation $z$. The nonlinear injection $\chi: \mathbb{R} \times \mathbb{R} \rightarrow \mathbb{R}^{n}$ takes the following structure:

$$
\chi\left(v_{1}, y-C z\right):=\left[\begin{array}{c}
k_{1}\left\lceil(y-C z)-v_{1}\right\rfloor^{\frac{n-1}{n}} \\
k_{2}\left\lceil(y-C z)-v_{1}\right\rfloor^{\frac{n-2}{n}} \\
\vdots \\
k_{n}\left\lceil(y-C z)-v_{1}\right\rfloor^{0}
\end{array}\right],
$$

with some positive gains $k_{i}, i=\overline{1, n}$. Finally, the matrix $A_{0}$ and the vector $a$ are given as follows

$$
A_{0}=\left[\begin{array}{ccccc}
0 & 1 & 0 & \cdots & 0 \\
0 & 0 & 1 & \cdots & 0 \\
& \vdots & & \ddots & \vdots \\
0 & 0 & 0 & 0 & 1 \\
0 & 0 & 0 & 0 & 0
\end{array}\right], a=\left[\begin{array}{c}
a_{1} \\
a_{2} \\
\vdots \\
a_{n}
\end{array}\right]
$$

where $a_{i}, i=\overline{1, n}$ are the vector of coefficients of the characteristic polynomial of the matrix $(A-L C)$. The solutions of the adaptive observer (7) are understood in the Filippov sense [26]. Let us introduce the following assumption:

Assumption 2. Let $0<\varrho_{\min } \leq \sigma_{\min }(C \Omega(t))$ for all $t \geq 0$ and $\|C \Omega\|_{\infty} \leq \varrho_{\max }<+\infty$.

The existence of $\varrho_{\max }$ is guaranteed by Assumption 1 and the fact that matrix $(A-L C)$ is Hurwitz; the existence of $\varrho_{\text {min }}$ follows if, for example, the number of sensors is equal to the number of unknown parameters, or $C \Omega(t)$ is uniformly injective for all $t \geq 0$ (for more details, see [27]). Moreover, note that $\Omega(0)$ can be arbitrarily chosen.

Remark 1. Opposite to [19] and [20], the adaptive observer in (7) strikes systems with relative degree larger than one. For instance, mechanical systems, where the relative degree of the output (position), with respect to the external disturbance $w(t)$ (in acceleration), is equal to two.

In the sequel, the stability of the HOSM adaptive observer (7) is analyzed. To this aim, the dynamics (7) can be rewritten as two interconnected subsystems given by the nonlinear parameter identification algorithm and the HOSM observer. Then, the FT-ISS properties of each subsystem are introduced. Finally, the small-gain theorem is used to ensure the stability of the adaptive estimation error.

\section{A. The Nonlinear Parameter Identification Algorithm}

Define $\tilde{\theta}:=\hat{\theta}-\theta$. Then, the error dynamics for $\tilde{\theta}$ is given by:

$$
\dot{\tilde{\theta}}=\Gamma \Omega^{T} C^{T}\lceil y-C \hat{x}\rfloor^{\alpha} .
$$

The FT-ISS convergence properties of the error dynamics (8) with respect to the input $\eta=T \delta-v$ with $\delta=x-z+\Omega \tilde{\theta}$, for $\alpha \in(0,1)$ and $\alpha=0$, are given by the following lemmas.
Lemma 2. [20]. Let Assumption 2 be satisfied. Then, the error dynamics (8), with $\alpha \in(0,1)$ and $\Gamma=\Gamma^{T}>0$, is FT-ISS with respect to $\eta$. Moreover, the trajectories satisfy:

$$
\begin{aligned}
& \begin{aligned}
&\left\|\tilde{\theta}\left(t, \tilde{\theta}_{0}\right)\right\| \leq \sqrt{c_{1}}\left(c_{2}^{\frac{\alpha-1}{2}}\left\|\tilde{\theta}_{0}\right\|^{1-\alpha}\right. \\
&\left.+\frac{\kappa_{1}(1-\psi)(1-\alpha) \varrho_{\min }^{\alpha+1} c_{2}^{\frac{\alpha+1}{2}}}{2} t\right)^{\frac{1}{1-\alpha}}, \forall t \leq T_{\tilde{\theta}_{1}}, \\
&\left\|\tilde{\theta}\left(t, \tilde{\theta}_{0}\right)\right\| \leq \sqrt{\frac{c_{1}}{c_{2}}} \mu_{\tilde{\theta}_{1}}\|\eta\|_{\infty}, \forall t>T_{\tilde{\theta}_{1}},
\end{aligned} \\
& \text { where } \tilde{\theta}_{0}=\tilde{\theta}(0), c_{1}:=2 \lambda_{\max }(\Gamma), c_{2}:=2 \lambda_{\min }(\Gamma), \kappa_{1} \in \\
& (0,1) \text { and }
\end{aligned}
$$

$$
\begin{gathered}
\mu_{\tilde{\theta}_{1}}=\frac{\left(2 \kappa_{2}\right)^{\frac{1}{\alpha+1}}\left\|C T^{-1}\right\|}{\left(\psi \kappa_{1}\right)^{\frac{1}{\alpha+1}} \varrho_{\min }}, \\
T_{\tilde{\theta}_{1}} \leq \max \left[0, \frac{2\left(c_{2}^{\frac{\alpha-1}{2}}\left\|\tilde{\theta}_{0}\right\|^{1-\alpha}-c_{1}^{\frac{\alpha-1}{2}} \mu_{\tilde{\theta}_{1}^{1-\alpha}}^{1-\alpha)}\right.}{\kappa_{1}(1-\psi)(1-\alpha) \varrho_{\min }^{\alpha+1} c_{2}^{\frac{\alpha+1}{2}}}\right],
\end{gathered}
$$

for any $\tilde{\theta}_{0} \in \mathbb{R}, \psi \in(0,1)$ and $\kappa_{2}=\max \left(\kappa_{1}+1, \kappa_{1} /(1-\right.$ $\left.\left.\kappa_{1}^{\frac{1}{\alpha}}\right)^{\alpha}\right)$.

Proof. Let us consider the following Lyapunov function:

$$
V_{\tilde{\theta}}=\frac{1}{2} \tilde{\theta}^{T} \Gamma^{-1} \tilde{\theta}
$$

that satisfies the following inequalities:

$$
\begin{gathered}
c_{1}^{-1}\|\tilde{\theta}\|^{2} \leq V_{\tilde{\theta}}(\tilde{\theta}) \leq c_{2}^{-1}\|\tilde{\theta}\|^{2} \\
c_{1}^{-\frac{\alpha+1}{2}}\|\tilde{\theta}\|^{\alpha+1} \leq V_{\tilde{\theta}}^{\frac{\alpha+1}{2}}(\tilde{\theta}) \leq c_{2}^{-\frac{\alpha+1}{2}}\|\tilde{\theta}\|^{\alpha+1} .
\end{gathered}
$$

Following the procedure given in [20], it can be shown that the time derivative of $V_{\tilde{\theta}}$ along the trajectories of the error dynamics (8), with $\alpha \in(0,1)$, satisfies

$$
\begin{aligned}
& \dot{V}_{\tilde{\theta}} \leq-\kappa_{1}(1-\psi) \varrho_{\min }^{\alpha+1} c_{2}^{\frac{\alpha+1}{2}} V_{\tilde{\theta}}^{\frac{\alpha+1}{2}}, \\
& \forall\|\tilde{\theta}\| \geq \frac{\left(2 \kappa_{2}\right)^{\frac{1}{\alpha+1}}\left\|C T^{-1}\right\|}{\left(\psi \kappa_{1}\right)^{\frac{1}{\alpha+1}} \varrho_{\min }}\|\eta\|_{\infty},
\end{aligned}
$$

for any constant $\psi \in(0,1)$. Therefore, the error dynamics (7b) is FT-ISS with respect to $\eta$.

Lemma 3. [20]. Let Assumption 2 be satisfied. Then, the error dynamics (8), with $\alpha=0$, is FT-ISS with respect to $\eta$. Moreover, the trajectories satisfy:

$$
\begin{aligned}
& \left\|\tilde{\theta}\left(t, \tilde{\theta}_{0}\right)\right\| \leq \sqrt{c_{1}}\left(c_{2}^{-\frac{1}{2}}\left\|\tilde{\theta}_{0}\right\|\right. \\
& \left.+\frac{\kappa_{1}(1-\psi) \varrho_{\min } c_{2}^{\frac{1}{2}}}{2} t\right), \forall t \leq T_{\tilde{\theta}_{2}}, \\
& \left\|\tilde{\theta}\left(t, \tilde{\theta}_{0}\right)\right\| \leq \sqrt{\frac{c_{1}}{c_{2}}} \mu_{\tilde{\theta}_{2}}\|\eta\|_{\infty}, \forall t>T_{\tilde{\theta}_{2}},
\end{aligned}
$$

where

$$
\begin{gathered}
\mu_{\tilde{\theta}_{2}}=\frac{2 \kappa_{2}\left\|C T^{-1}\right\|}{\psi \kappa_{1} \varrho_{\min }}, \\
T_{\tilde{\theta}_{2}} \leq \max \left[0, \frac{2\left(c_{2}^{-\frac{1}{2}}\left\|\tilde{\theta}_{0}\right\|-c_{1}^{-\frac{1}{2}} \mu_{\tilde{\theta}_{2}}\right)}{\kappa_{1}(1-\psi) \varrho_{\min } c_{2}^{\frac{1}{2}}}\right],
\end{gathered}
$$


for any $\tilde{\theta}_{0} \in \mathbb{R}, \psi \in(0,1)$ and $\kappa_{2} \geq \kappa_{1}+1$.

Proof. Let us consider the Lyapunov function given in (9) that satisfies the inequalities (10) and (11). Following the procedure given in [20], the time derivative of $V_{\tilde{\theta}}$ along the trajectories of the error dynamics (8), with $\alpha=0$, satisfies

$$
\begin{gathered}
\dot{V}_{\tilde{\theta}} \leq-\kappa_{1}(1-\psi) \varrho_{\min } c_{2}^{\frac{1}{2}} V_{\tilde{\theta}}^{\frac{1}{2}}, \\
\forall\|\tilde{\theta}\| \geq \frac{2 \kappa_{2}|| C T^{-1} \|}{\psi \kappa_{1} \varrho_{\min }}\|\eta\|_{\infty},
\end{gathered}
$$

for any constant $\psi \in(0,1)$. Therefore, the error dynamics (7b) is FT-ISS with respect to $\eta$.

Then, the results of Lemmas 2 and 3 establish that the trajectories of the error dynamics (8), i.e., $\tilde{\theta}$, enter into the bound $\sqrt{c_{1} / c_{2}} \mu_{\tilde{\theta}_{1}}$ or $\sqrt{c_{1} / c_{2}} \mu_{\tilde{\theta}_{2}}$, in a finite time for any $\alpha \in[0,1)$. It is clear that the size of such a bound depends on $\alpha$.

\section{B. The HOSM State Observer}

Consider the error dynamics for $\delta=x-z+\Omega \tilde{\theta}$, i.e.,

$$
\begin{aligned}
\dot{\delta} & =(A-L C) \delta+D w(t), \\
y_{\delta} & =y-C z=C \delta-C \Omega \tilde{\theta},
\end{aligned}
$$

where $y_{\delta}$ is a measurable signal. Then, applying the transformation $\bar{\delta}=T \delta$ to system (14), it follows that

$$
\begin{aligned}
\dot{\bar{\delta}} & =A_{0} \bar{\delta}+a C T^{-1} \bar{\delta}-a C \Omega \tilde{\theta}+T D w, \\
y_{\delta} & =\bar{\delta}_{1}-C \Omega \tilde{\theta},
\end{aligned}
$$

where $\bar{\delta}_{1}=C T^{-1} \bar{\delta}$ and $T D=\left[0, \ldots, 0, C A^{n-1} D\right]^{T}$. Then, an observer for system (15) is designed as (7e). Therefore, the error dynamics for $\eta=\bar{\delta}-v$ is given by

$$
\dot{\eta}=A_{0} \eta+T D w-\chi\left(v_{1}, y-C z\right) .
$$

Note that $y-C z-v_{1}=\bar{\delta}_{1}-C \Omega \tilde{\theta}-v_{1}$; therefore, the correction term $\chi_{1}$ can be rewritten as follows

$$
\chi\left(\eta_{1}, C \Omega \tilde{\theta}\right)=\left[\begin{array}{c}
k_{1}\left[\eta_{1}-C \Omega \tilde{\theta}\right]^{\frac{n-1}{n}} \\
k_{2}\left[\eta_{1}-C \Omega \tilde{\theta}\right]^{\frac{n-2}{n}} \\
\vdots \\
k_{n}\left[\eta_{1}-C \Omega \tilde{\theta}\right]^{0}
\end{array}\right],
$$

where $\eta_{1}=\bar{\delta}_{1}-v_{1}$. Then, the error dynamics is given by

$$
\dot{\eta}=A_{0} \eta+T D w(t)-\chi\left(\eta_{1}, C \Omega \tilde{\theta}\right)
$$

Note that dynamics (16) has the same structure as the HOSM differentiator [28]. The following lemma states the FTISS convergence properties of the error dynamics (16) with respect to the input $\tilde{\theta}$.

Lemma 4. [28], [29]. Let the observer (7c)-(7e) be applied to the system (1). Let Assumption 1 be satisfied. Then, there exist some positive constants $k_{i}, i=\overline{1, n}$, such that the estimation error dynamics (16) is FT-ISS with respect to $\tilde{\theta}$.
According to [28], if $\|w\|_{\infty} \leq \bar{w}$, with some known positive constant $\bar{w}$; then, the gains $k_{i}, i=\overline{1, n}$, for $n=4$, can be chosen as $k_{4}=1.1\left|C A^{n-1} D \bar{w}\right|, k_{3}=1.5\left|C A^{n-1} D \bar{w}\right|^{1 / 2}$, $k_{2}=2\left|C A^{n-1} D \bar{w}\right|^{1 / 3}$ and $k_{1}=3\left|C A^{n-1} D \bar{w}\right|^{1 / 4}$.

Moreover, the results given by [28] show that $\eta$ satisfies the following inequality

$$
\left\|\eta\left(t, \eta_{0}\right)\right\| \leq \sum_{i=1}^{n} \tau_{i}\left|C A^{n-1} D \bar{w}\right|^{\frac{i-1}{n}} \varrho \frac{n-i+1}{n}\|\tilde{\theta}\|_{\infty^{\frac{n-i+1}{n}}}^{\frac{n-1}{n}},
$$

for all $t \geq T_{\eta}>0$, with $\tau_{i} \geq 1$ depending only on the gains $k_{i}$. A method to compute the constants $\tau_{i}$ has been proposed in [29], by means of the following Lyapunov function

$$
V_{\eta}=\sum_{j=1}^{n-1} \beta_{j} Z_{j}\left(\eta_{j}, \eta_{j+1}\right)+\beta_{n} \frac{1}{p}\left|\eta_{n}\right|^{p},
$$

with some $\beta_{i}>0, i=\overline{1, n}, p>1$ and $Z_{j}\left(\eta_{j}, \eta_{j+1}\right)=$ $\frac{r_{j}}{p}\left|\eta_{j}\right|^{\frac{p}{r_{j}}}-\eta_{j}\left\lceil\eta_{j+1}\right\rfloor^{\frac{p-r_{j}}{r_{j+1}}}+\left(\frac{p-r_{j}}{p}\right)\left|\eta_{j+1}\right|^{\frac{p}{r_{j+1}}}, j=\overline{1, n-1}$, and the homogeneity weights $r_{i}=n+1-i$ with $i=\overline{1, n}$.

Hence, note that by definition $\hat{x}=z+T^{-1} v$; thus, since $\delta=x-z+\Omega \tilde{\theta}$ and $\eta=T \delta-v$, one obtains that

$$
x-\hat{x}=T^{-1} \eta-\Omega \tilde{\theta},
$$

then, Lemma 4 implies that the state estimation error dynamics $x-\hat{x}$ is FT-ISS with respect to $\tilde{\theta}$.

\section{Convergence of the Adaptive Observer}

In order to establish the convergence properties of the adaptive observer (6), the statements provided by Lemmas 2 , 3, 4 and Theorem 1 are applied. The following result shows that the interconnected error system (8) and (16) is GUS for any $\alpha \in[0,1)$.

Theorem 2. Let Assumptions 1 and 2 hold. Let $L \in \mathbb{R}^{n}$ be such that $(A-L C)$ is Hurwitz, $\alpha \in[0,1), \Gamma=\Gamma^{T}>0$, and $k_{i}, i=\overline{1, n}$, be designed based on the formulas provided by [28]. If the following conditions

$$
\begin{gathered}
n \beta \mu_{\max } \leq 1, \\
{\left[(n \beta)^{n} \mu_{\max }\right]^{\frac{1}{n-1}} \leq 1,}
\end{gathered}
$$

hold with $\beta=\max _{i=\overline{1, n}}\left[\tau_{i}\left|C A^{n-1} D \bar{w}\right|^{\frac{i-1}{n}} \varrho_{\max }^{\frac{n-i+1}{n}}\right]$ and $\mu_{\max }=\sqrt{c_{1} / c_{2}} \max \left[\mu_{\tilde{\theta}_{1}}, \mu_{\tilde{\theta}_{2}}\right]$; then, the interconnected error system (8) and (16) is practically GUAS for any $\alpha \in[0,1)$.

Proof. Based on Lemmas 2, 3 and 4 and their proofs, considering (12b), (13b), and (17), it follows that

$$
\begin{gathered}
\|\tilde{\theta}\| \geq \mu_{\max }\|\eta\| \Rightarrow \dot{V}_{\tilde{\theta}}<0, \\
\sum_{i=1}^{n}\|\tilde{\theta}\|^{\frac{n-i+1}{n}} \leq \beta^{-1}\|\eta\| \Rightarrow \dot{V}_{\eta}<0,
\end{gathered}
$$

with $\mu_{\max }=\sqrt{c_{1} / c_{2}} \max \left[\mu_{\tilde{\theta}_{1}}, \mu_{\tilde{\theta}_{2}}\right]$ and

$$
\begin{gathered}
\mu_{\tilde{\theta}_{1}}=\frac{\left(2 \kappa_{2}\right)^{\frac{1}{\alpha+1}}|| C T^{-1} \|}{\left(\psi \kappa_{1}\right)^{\frac{1}{\alpha+1}} \varrho_{\min }}, \mu_{\tilde{\theta}_{2}}=\frac{2 \kappa_{2}\left\|C T^{-1}\right\|}{\psi \kappa_{1} \varrho_{\min }}, \\
\beta=\max _{i=\overline{1, n}}\left[\tau_{i}\left|C A^{n-1} D \bar{w}\right|^{\frac{i-1}{n}} \varrho_{\max }^{\frac{n-i+1}{n}}\right] .
\end{gathered}
$$


Note that (22) can be rewritten as follows

$$
\begin{aligned}
n\|\tilde{\theta}\| & \leq \beta^{-1}\|\eta\| \Rightarrow \dot{V}_{\eta}<0, \text { for }\|\tilde{\theta}\|>1, \\
n\|\tilde{\theta}\|^{\frac{1}{n}} \leq \beta^{-1}\|\eta\| & \Rightarrow \dot{V}_{\eta}<0, \text { for }\|\tilde{\theta}\| \leq 1 .
\end{aligned}
$$

Therefore, in order to apply the Theorem 1 , in terms of the error norms, two cases must be analyzed, i.e., when $\|\tilde{\theta}\|>1$ and when $\|\tilde{\theta}\| \leq 1$.

- Case 1: $s>1$

According to (21) and (22), the small-gain condition is reduced to $\mu_{\max } s \leq(n \beta)^{-1} s$, which is satisfied for any $s>1$, the previous condition can be rewritten as in (20a).

- Case 2: $s \leq 1$

According to (21) and (22), the small-gain condition is reduced to $\mu_{\max } s \leq(n \beta)^{-1} s^{n}$, which results in the condition given in (20b).

Thus, in order to guarantee the practical, global, and uniform asymptotic stability of the interconnected error systems (8) and (16), conditions (20a) and (20b) are required. This concludes the proof.

Theorem 2 implies that the state estimation error dynamics, $x-\hat{x}$; and the parameter identification error dynamics, $\tilde{\theta}$; are practically GUAS for any $\alpha \in[0,1)$.

The procedure to verify the condition (20) includes the following steps:

1) Use (7a) to calculate $0<\varrho_{\min } \leq \sigma_{\min }(C \Omega(t))$ and $\|C \Omega\|_{\infty} \leq \varrho_{\max }<+\infty$ offline.

2) Fix the values of $\kappa_{1}, \psi \in(0,1)$, and $\kappa_{2}$ according to Lemma 2 or 3.

3) Define the set $\mathcal{L}_{c}=\left\{\eta \in \mathbb{R}^{n} \mid V_{\eta} \leq c\right\}$, based on $V_{\eta}$ given in (18), with $c$ such that $\dot{V}_{\eta}<0$ in the sublevel set $\mathcal{L}_{c}$; and then, from the sublevel set $\mathcal{L}_{c}$, the values for $\tau_{i}$ can be computed as $\tau_{i}=\max _{\mathcal{L}_{c}}\left|\eta_{i}\right|$, for all $i=\overline{1, n}$.

4) Verify the small-gain condition (20).

Note that the values for $\varrho_{\min }$ and $\varrho_{\max }$ depend on the excitation given by the term $G(t, y, u)$ in (7a), which depends on the input $u$. It is well-known, in the adaptive estimation framework, that the input can be modified in order to get more excitation if it is required. Thus, it is possible to modify both $\varrho_{\min }$ and $\varrho_{\max }$ by means of the input. Note also that $\kappa_{1}, \kappa_{2}$ and $\psi$ can be adjusted to manipulate the left-hand side of (20).

The following properties for the adaptive observer (7) must be remarked:

1) In the case that inequalities (20a) and (20b) are not satisfied simultaneously, the results from the adaptive observer will be local if (20b) holds. Moreover, if (20b) is not satisfied but (20a) is, the interconnected error dynamics converges to a unitary neighborhood of the origin. Note that, if (20b) holds, the size of the error convergence region is characterized by $\left[(n \beta)^{n} \mu_{\max }\right]^{\frac{1}{n-1}}$.

2) Theorem 2 provides practical, global, and uniform asymptotic stability of the adaptive estimation error, and the rate of convergence given by the HOSM adaptive observer is faster than exponential due to its FT-ISS properties.

3) For the multiple-output and multiple-parameter case, consider system (1) with $y \in \mathbb{R}^{p}, \theta \in \mathbb{R}^{q}$ and $w \in \mathbb{R}^{p}$ with $q \leq p$, let us assume that the output vector has a vector relative degree $\left[r_{1}, r_{2}, \ldots, r_{p}\right]$ with respect to the unknown input. Therefore, if the relative degrees are such that $r_{1}+r_{2}+\ldots+r_{p}=n$; then, there exists a required nonsingular matrix $T \in \mathbb{R}^{n \times n}$ obtained from the rearrangement of the $n$ linearly independent rows of the observability matrix of the pair $(A, C)$. Under this vector relative degree condition, the HOSM state observer (7e) can be designed as follows

$$
\dot{v}_{j}=A_{j 0} v_{j}+A_{j 1}\left(y_{j}-c_{j} z\right)+\chi_{j}\left(v_{j 1}, y_{j}-c_{j} z\right),
$$

for $j=\overline{1, p}$, with

$$
\begin{gathered}
A_{j 0}=\left[\begin{array}{ccccc}
0 & 1 & 0 & \cdots & 0 \\
0 & 0 & 1 & \cdots & 0 \\
& \vdots & & \ddots & \vdots \\
0 & 0 & 0 & 0 & 1 \\
0 & 0 & 0 & 0 & 0
\end{array}\right]_{r_{j} \times r_{j}}, A_{j 1}=\left[\begin{array}{c}
a_{j 1} \\
a_{j 2} \\
\vdots \\
a_{j r_{j}}
\end{array}\right]_{r_{j}}, \\
c_{j}=\left[\begin{array}{c}
1 \\
0 \\
\vdots \\
0
\end{array}\right]_{r_{j}}^{T}, \chi_{j}=\left[\begin{array}{c}
k_{j 1}\left\lceil\left(y_{j}-c_{j} z\right)-v_{j 1}\right\rfloor^{\frac{r_{j}-1}{r_{j}}} \\
k_{j 2}\left\lceil\left(y_{j}-c_{j} z\right)-v_{j 1}\right\rfloor^{\frac{r_{j}-2}{r_{j}}} \\
\vdots \\
k_{j r_{j}}\left\lceil\left(y_{j}-c_{j} z\right)-v_{j 1}\right]^{0}
\end{array}\right],
\end{gathered}
$$

where $v=\left[v_{1}, \ldots, v_{p}\right] \in \mathbb{R}^{n}$, with $v_{j}=\left[v_{j 1}, \ldots v_{j r_{j}}\right] \in$ $\mathbb{R}^{r_{j}}$, and some constant vectors $A_{j 1}$, for all $j=\overline{1, p}$. Then, it is possible to apply the HOSM observers $v_{j}$ by diagonal output blocks, obtaining a small-gain condition for each output block. Thus, if such smallgain conditions hold, it is possible to provide a state estimation and a parameter identification simultaneously (for more details, see [30] and [31]).

4) For the case of time-varying parameters, i.e., $\theta(t)$, the proposed parameter identification algorithm provides the same result, i.e., practically GUAS. However, based on [20], it is possible to prove that the size of the convergence region also depends on the upper bound of the time-varying parameter.

5) The robustness and finite-time properties of the HOSM observer together with the finite-time parameter identification algorithm are illustrated in the next section and, in contrast with [13], [15], [18], [19] and [20], the proposed algorithm covers a broader class of systems.

\section{Simulation Results}

The simulations have been done in Matlab Simulink with the Euler explicit discretization method and sampling time equal to 0.001 [s].

Consider the DC motor system [32]:

$$
\begin{array}{r}
\dot{x}=\left[\begin{array}{ccc}
-\frac{R_{a}}{L_{a}} & -\frac{K_{m}}{L_{a}}-B_{s} & B_{s} \\
\frac{K_{m}}{J_{m}} & 0 & 0 \\
0 & B_{s} & -\frac{B_{l}}{J_{l}}-B_{s}
\end{array}\right] x+\left[\begin{array}{c}
\frac{1}{L_{a}} u \\
-T_{f}\left(x_{2}\right) \\
0
\end{array}\right] \\
+\left[\begin{array}{c}
0 \\
-\frac{1}{J_{m}} x_{2} \\
0
\end{array}\right] \theta+\left[\begin{array}{l}
0 \\
0 \\
1
\end{array}\right] w(t),
\end{array}
$$

$y=\left[\begin{array}{lll}0 & 1 & 0\end{array}\right] x+\varsigma$, 
where $x=\left[x_{1}, x_{2}, x_{3}\right]^{T}, x_{1} \in \mathbb{R}$ is the armature current, $x_{2} \in$ $\mathbb{R}$ is the rotor angular position, and $x_{3} \in \mathbb{R}$ is the rotor angular velocity. The parameters are: $L_{a}=0.21[\mathrm{H}]$ the armature inductance, $R_{a}=10[\Omega]$ the armature resistance, $K_{m}=3$ the torque constant, $B_{s}=0.18[\mathrm{Nm} /(\mathrm{rad} / \mathrm{s})]$ the inner damping coefficient of the shaft, $J_{m}=19\left[\mathrm{Kgm}^{2}\right]$ the motor inertia moment, $J_{l}=12.5\left[\mathrm{Kgm}^{2}\right]$ the load inertia moment, and $B_{l}=$ $3.4[\mathrm{Nms} / \mathrm{rad}]$ the load viscous friction. The nonlinear function $T_{f}$ represents a mathematical model of the friction-velocity curve representing the transition from static to kinetic friction, including the Coulomb friction, and it is given as

$$
T_{f}(q)=\alpha_{0 q} \operatorname{sign}(q)+\alpha_{1 q} e^{-\alpha_{2 q} q|q|} \operatorname{sign}(q),
$$

for any $q \in \mathbb{R}$ and some known coefficients $\alpha_{0 x_{2}}=3.2$, $\alpha_{1 x_{2}}=2.4$ and $\alpha_{2 x_{2}}=1.1$.

The external disturbance is given by $w(t)=-T_{d}-T_{f}\left(x_{3}\right)$, where $T_{d}$ is the load disturbance torque. For simulation purposes, the load disturbance torque is taken as $T_{d}=0.4$ $[\mathrm{Nm}]$, and the friction coefficients as $\alpha_{0 x_{3}}=1.1, \alpha_{1 x_{3}}=2.1$, and $\alpha_{2 x_{3}}=0.75$. Thus, the external disturbance can be upper bounded by $\|w\|_{\infty} \leq 3.6$.

The parameter to be identified is the motor viscous friction given by $\theta=4.6[\mathrm{Nms} / \mathrm{rad}]$. The input is given as $u(t)=$ $3 \sin (t)+1.4 \sin (0.45 t)+8 \cos (5 t)$.

The proposed algorithm is compared with the linear algorithm, i.e., with $\alpha=1$. Note that the adaptive observers [13], [15], [18], [19] and [20] cannot be applied because the relative degree of the output, with respect to the external disturbance, is equal to three. Moreover, measurement noise is considered and is denoted by $\varsigma$, which is a band limited white noise with power of $1 \times 10^{-7}$.

Consider the initial conditions $x(0)=[-0.2,-0.1,1]^{T}$ while the observer initial conditions are taken as $\Omega(0)=0.1$, $\hat{\theta}(0)=0, z(0)=0$ and $v(0)=0$. Fixing $\Gamma=140$, $k_{1}=12.96, k_{2}=2.84$ and $k_{3}=3.96$ selected as [28] with $\bar{w}=3.6$. Also, the transformation matrix $T$ and the vector $a$ are given as follows

$$
T=\left[\begin{array}{ccc}
-304.4 & 6.3 & 0 \\
1 & 0 & 0 \\
7.1 & -15.9 & 35.1
\end{array}\right], a=\left[\begin{array}{c}
-15 \\
-71 \\
-105
\end{array}\right]
$$

The Assumption 2 is satisfied with $\varrho_{\min }=0.024$ and $\varrho_{\max }=0.045$. Fixing $\kappa_{1}=0.99, \kappa_{2}=1.99, \psi=0.99$ and using the method described by [29], it can be shown that $\tau_{1}=0.52, \tau_{2}=0.12$ and $\tau_{3}=0.18$. Then, it is easy to verify that all the conditions of Theorem 2 are satisfied for all $\alpha \in[0,1)$. Fig. 1 illustrates the convergence of the observer to the states of the system in the presence of measurement noise. Fig. 2 shows the estimation of the parameter for different values of $\alpha$. It has to be remarked that the nonlinear and the discontinuous adaptive observers, i.e., $\alpha \in[0,1)$, converge to a neighborhood of the origin even in the presence of disturbances, and the convergence rate is faster than the linear algorithm, i.e., $\alpha=1$.

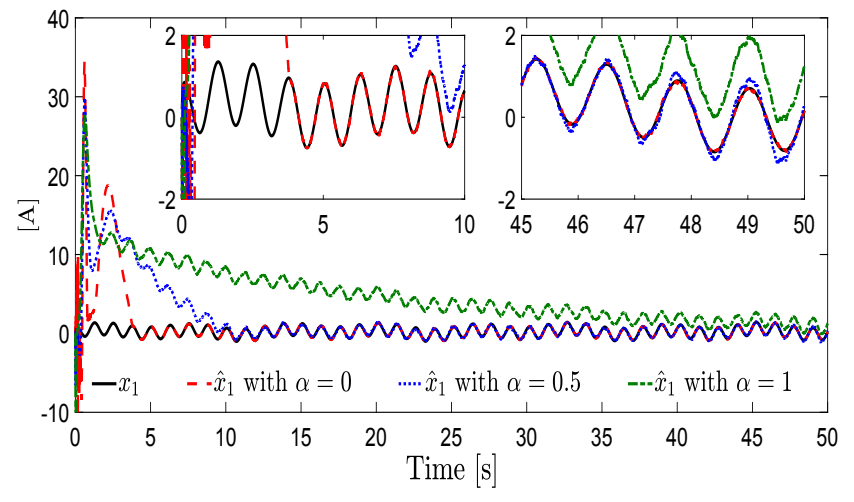

(a) The state $x_{1}$ and the estimated signal $\hat{x}_{1}$.

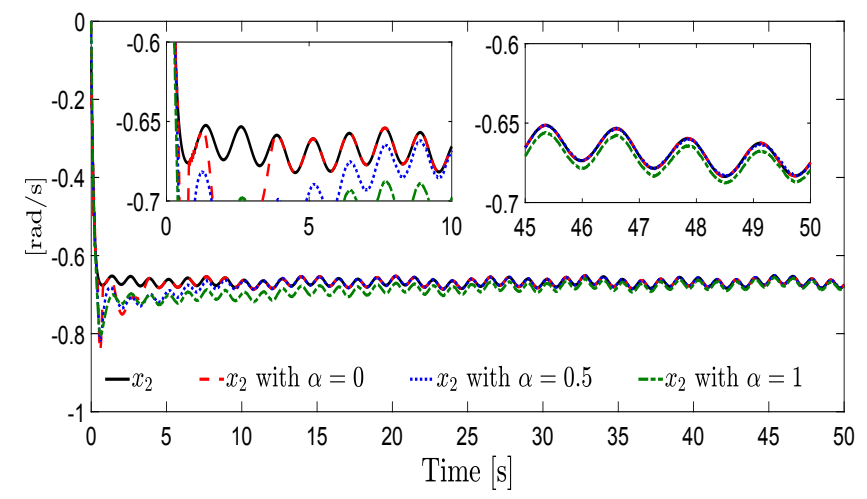

(b) The state $x_{2}$ and the estimated signal $\hat{x}_{2}$

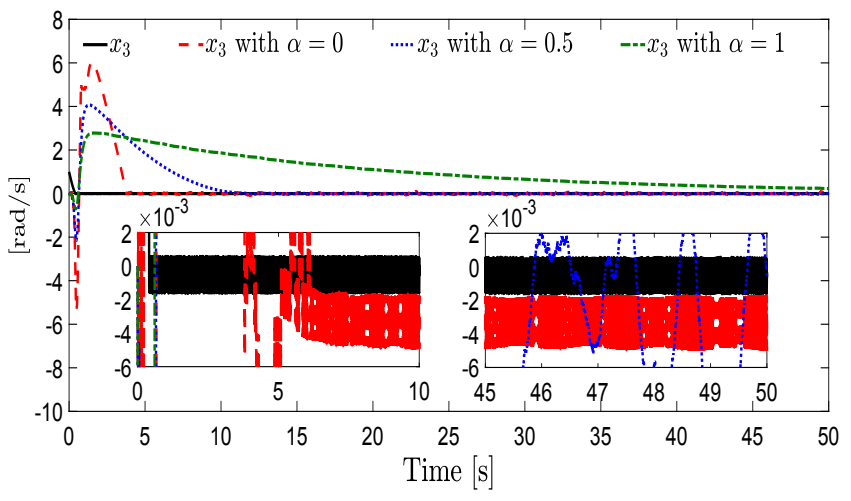

(c) The state $x_{3}$ and the estimated signal $\hat{x}_{3}$.

Figure 1: States behavior and estimated states

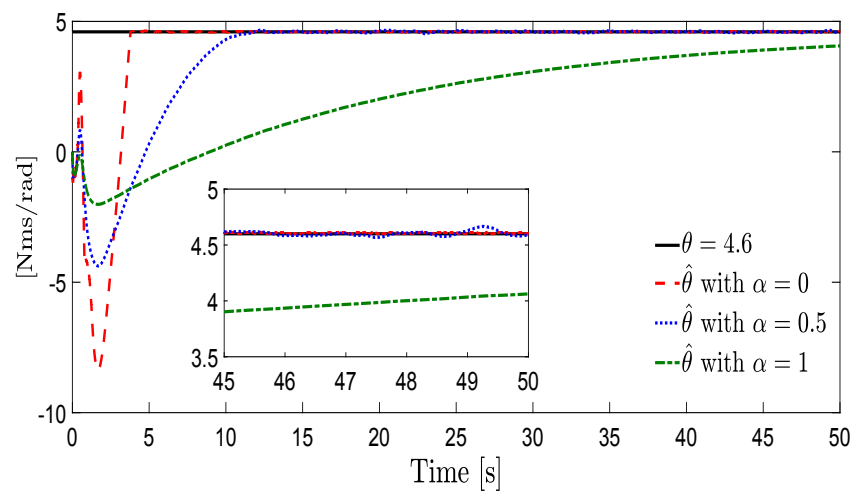

Figure 2: The parameter $\theta$ and the identified parameter $\hat{\theta}$. 
To illustrate the convergence of algorithm, the following error index is proposed $e_{R M S}(t)=$ $\left(\frac{1}{\Delta T} \int_{t-\Delta t}^{t}\|\tilde{\theta}(\tau), e(\tau)\|^{2} d \tau\right)^{\frac{1}{2}}$, where $\Delta T=2$ is a time window width. The results are depicted by Fig. 3

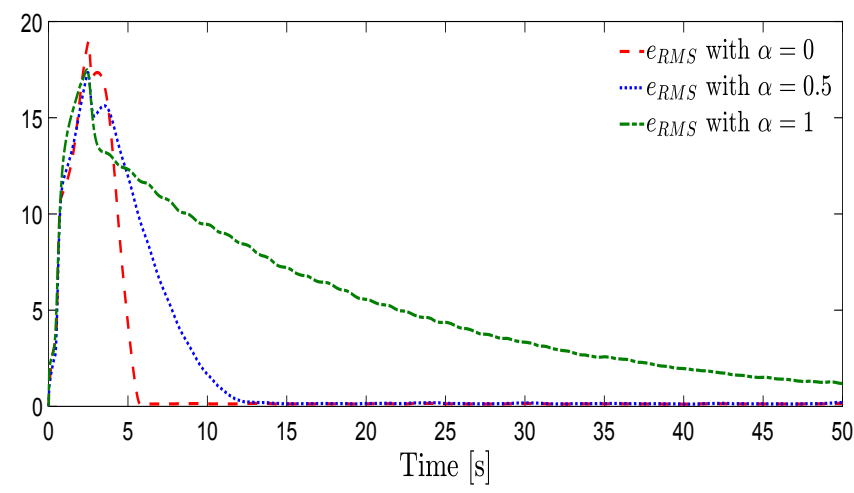

Figure 3: Estimation error index.

It is clear that the algorithm with $\alpha=0$ provides the best performance.

\section{CONCLUSiOnS}

This paper contributes with an HOSM adaptive observer for a class of uncertain nonlinear systems with external disturbances. The disturbances may have a relative degree higher than one in contrast with previous works (see, for instance, [19]-[20]). The proposed approach combines an HOSM observer with a nonlinear parameter identification algorithm. The HOSM observer guarantees FT-ISS of the state estimation error with respect to the parameter identification error. The nonlinear parameter identification algorithm provides FT-ISS of the parameter identification error with respect to the state estimation error. Then, the practical, global and uniform asymptotic stability of the adaptive estimation error, despite the external disturbances, is guaranteed through the small-gain theorem. Simulation results illustrate the performance of the proposed HOSM adaptive observer.

\section{REFERENCES}

[1] P. A. Ioannou and J. Sun, Robust Adaptive Control. New Jersey, USA: Prentice Hall, Inc., 1996.

[2] Y. Shtessel, C. Edwards, L. Fridman, and A. Levant, Sliding Mode Control and Observation. New York: Birkhauser, 2014.

[3] G. Besançon, Nonlinear Observers and Application. Springer-Verlang Berlin Heidelberg, 2007.

[4] R. Marino and P. Tomei, Nonlinear Control Design: Geometric, Adaptive \& Robust. London, Prentice Hall, 1995.

[5] Y. M. Cho and R. Rajamani, "A systematic approach to adaptive observer synthesis for nonlinear systems," IEEE Transactions on Automatic Control, vol. 42, no. 4, pp. 534-537, 1997.

[6] M. Ekramian, F. Sheikholeslam, S. Hosseinnia, and M. Yazdanpanah, "Adaptive state observer for Lipschitz nonlinear systems," Systems \& Control Letters, vol. 62, pp. 319-323, 2013.

[7] I. Tyukin, E. Steur, H. Neijmeijer, and C. Leuwen, "Adaptive observers and parameter estimation for a class of systems nonlinear in the parameters," Automatica, vol. 49, p. 2409.2423, 2013.

[8] M. Farza, M. Saad, T. Maatoug, and M. Kamoun, "Adaptive observers for nonlinearly parametrized class of nonlinear systems," Automatica, vol. 45, pp. 2292-2299, 2009.
[9] T. Ma and C. Cao, "Estimation using $L_{1}$ adaptive descriptor observer for multivariable systems with nonlinear uncertainties and measurement noises," European Journal of Control, pp. 1-8, 2019. DOI: https://doi.org/10.1016/j.ejcon.2019.08.007.

[10] V. Stepanyan and N. Hovakimyan, "Robust adaptive observer design for uncertain systems with bounded disturbances," IEEE Transactions on Neural Networks, vol. 18, pp. 1392-1403, 2007.

[11] P. Vijay, M. Tade, and Z. Shao, "Adaptive observer based approach for the fault diagnosis in solid oxide fuel cells," Journal of Process Control, vol. 84, pp. 101-114, 2019.

[12] L. Chen, P. Shi, and M. Liu, "Fault reconstruction for markovian jump systems with iterative adaptive observer," Automatica, vol. 105, pp. 254263, 2019.

[13] M. Farza, M. Saad, T. Menard, A. Ltaief, and T. Maatoug, "Adaptive observer design for a class of nonlinear systems: Application to speed sensorlees induction motor," Automatica, vol. 90, pp. 239-247, 2018.

[14] H. Rabiee, M. Ataei, and M. Ekramian, "Continuous nonsingular terminal sliding-mode control based on adaptive sliding-mode disturbance observer for uncertain nonlinear systems," Automatica, vol. 109, p. 108515, 2019.

[15] Z. Chen, M. Tomita, S. Doki, and S. Okuma, "New adaptive sliding observers for position and velocity-sensorless controls of brushless dc motors," IEEE Transactions on Industrial Electronics, vol. 47, pp. 582$591,2000$.

[16] M. Angulo, J. A. Moreno, and L. Fridman, "Robust exact uniformly convergent arbitrary order differentiator," Automatica, vol. 49, no. 8, pp. 2489-2495, 2013

[17] M. Angulo, J. Moreno, and L. Fridman, "On functional observers for linear systems with unknown inputs and HOSM differentiators," Journal of the Franklin Institute, vol. 351, pp. 1982-1994, 2014.

[18] A. Pyrkin, A. Bobtsov, R. Ortega, and A. Vedyakov, "Adaptive state observers using dynamic regressor extension and mixing," Systems \& Control Letters, vol. 133, p. 104519, 2019.

[19] H. Ríos, D. Efimov, and W. Perruqueti, "An adaptive sliding-mode observer for a class of uncertain nonlinear systems," International Journal of Adaptive Control and Signal Processing, vol. 32, pp. 511527, 2018.

[20] R. Franco, H. Rios, D. Efimov, and W. Perruquetti, "Adaptive estimation for uncertain nonlinear systems with measurement noise: A sliding-mode observer approach," International Journal of Robust and Nonlinear Control, vol. 31, pp. 3809-3826, 2020.

[21] E. Bernuau, A. Polyakov, D. Efimov, and W. Perruqueti, "Verification of ISS, iISS and IOSS properties applying weighted homogeneity," System \& Control Letters, vol. 62, pp. 1159-1167, 2013.

[22] Z. Jiang, I. Mareels, and Y. Wang, "A Lyapunov formulation of the nonlinear small-gain theorem for interconnected ISS systems," Automatica, vol. 32, pp. 1211-1215, 1996.

[23] H. K. Khalil, Nonlinear Systems. Prentice Hall, third ed., 2002.

[24] Z. Jiang, A. Teel, and L. Praly, "Small-gain theorem for ISS systems and applications," Mathematics and Control Signals Systems, vol. 7, pp. $92-120,1994$

[25] S. Dashkovskiy, B. Ruffer, and F. Wirth, "An ISS small-gain theorem for general networks," Mathematics and Control Signals Systems, vol. 19, pp. 93-122, 2007.

[26] A. Filippov, Differential Equations with Discontinuos Right-hand Sides. Dordrecht, The Netherlands: Kluwer Academic Publishers, 1988.

[27] J. Rueda-Escobedo, J. Moreno, and J. Schiffer, "Discontinuous gradient algorithm for finite-time estimation of time-varying parameters," International Journal of Control, vol. 89, pp. 1838-1848, 2016.

[28] A. Levant, "High-order sliding modes: differentiation and outputfeedback control," International Journal of Control, vol. 76, no. 9-10, pp. 924-941, 2003.

[29] E. Zavala and J. Moreno, "Levant's arbitrary-order exact differentiator: A Lyapunov approach," IEEE Transactions on Automatic Control, vol. 64 , pp. 3034-3039, 2019.

[30] L. Fridman, A. Levant, and J. Davila, "Observation of linear systems with unknown inputs via high-order sliding-modes," International Journal of System Science, vol. 38, no. 10, pp. 773-791, 2007.

[31] H. Ríos, M. Mera, D. Efimov, and A. Polyakov, "Robust output-control for uncertain linear systems: Homogeneous differentiator-based observer approach," International Journal of Robust and Nonlinear Control, vol. 27, no. 11, pp. 1895-1924, 2017.

[32] M. Dormin and P. Gutman, "Controlling mechanical systems with backlash: A survey," Automatica, vol. 38, pp. 1633-1649, 2002. 